\title{
USE OF PARTIAL DERIVATIVES TO DERIVE A CONVERGENT NUMERICAL SCHEME WITH ITS ERROR ESTIMATES
}

\author{
Sania Qureshi ${ }^{1}$, Oluwaseun Adeyeye ${ }^{2} \&$ Asif Ali Shaikh ${ }^{1}$ \\ ${ }^{1}$ Department of Basic Sciences and Related Studies, Mehran University of Engineering \\ and Technology, Jamshoro 76062, Pakistan \\ ${ }^{2}$ Department of Mathematics, School of Quantitative Sciences, Universiti Utara Malaysia, Sintok \\ 06010, Kedah, Malaysia \\ sania.qureshi@faculty.muet.edu.pk,adeyeye@uum.edu.my,asif.shaikh@faculty.muet.edu.pk
}

Received: 22 September 2019; Accepted: 17 December 2019

\begin{abstract}
Using the idea of the partial derivative with respect to the ordinate of a given mathematical function, a new numerical scheme having third order convergence has been devised for solving initial value problems in ordinary differential equations. Such problems are deemed to be indispensable in diverse fields of science, medical and engineering and are most often required to be solved by the numerical schemes. In view of this, the proposed numerical scheme is found to be efficient in solving both autonomous and non-autonomous type of problems as supported by some numerical experiments in the present study. Using the Taylor expansion for the slopes involved in the scheme, the leading term of the local truncation error is shown to have contained $\mathscr{O}\left(h^{4}\right)$ which proves third order accuracy of the scheme. In addition to this, consistency and linear stability analysis of the proposed scheme has extensively been discussed. Numerical experiments show better performance of the proposed numerical scheme when compared with existing numerical schemes of the same order as that of the scheme proposed. CPU time (seconds), maximum absolute relative error and the absolute relative error, computed at the last grid point of the integration interval for the associated initial value problem, are the parameters to test the performance of the proposed numerical scheme. MATLAB Version: 9.4.0.813654 (R2018a) in double-precision on a personal computer equipped with a Processor Intel (R) Core(TM) i3-4500U CPU@ $1.70 \mathrm{GHz}$ running under the Windows 10 operating system has been employed in order to carry out all the required numerical computations.
\end{abstract}

MSC 2010: 26A33, $34 M 03$

Keywords: multi-derivative, local truncation error, stability, absolute relative errors, consistency, principal term

\section{Introduction}

Initial value problems in ordinary differential equations have always been a major concern for many mathematicians and physicists related to different fields of study. This is so because the initial value problems have played an important role in modeling very important physical laws of nature. The theory of differential equations 
is used to understand the marginal analysis in economics, motion of planets in the solar system, RL, RC and RLC series circuits in electrical circuits, laws of Newton in classical mechanics, rotation of a fluid in computational fluid dynamics, mass-spring system in mechanical engineering, volume in the single compartmental lung in the clinical medicine, drainage of a liquid from a container in hydrodynamics, snowplow model in dynamics, designing a solar collector, Lotka-Volterra model in population dynamics, mixing problems in chemistry, heating and cooling of buildings, Stefan's law of radiation, spread of a disease in medical science, chemical reactions, deflection of a beam in civil engineering, nonlinear pendulum in mechanics, forgetfulness and theory of learning and many more, (see for example [1-9]). Thus, the study of differential equations complete the understanding of many fields indispensable to mathematicians, engineers, physicists, businessmen, medical professionals; just to name a few.

Despite the frequent occurrence of these mathematical models in the number of interesting areas, it cannot be denied that most of these models are not exactly solvable mainly due to the involvement of nonlinear terms and stiffness of the problem in particular [10]. In other words, their solutions can neither be represented in terms of elementary mathematical functions nor can they be found. This is where numerical techniques come to our rescue. With the advent of digital computers, it has now become extremely simple to get accurate approximate solutions to mathematical models once considered to be unfathomable. Instead of producing a closed form solution for a model, numerical techniques generate a sequence of results in a discrete fashion which is easily tabulated for graphical interpretation of the solution. One mathematical model is different from other in many respects such as physical interpretation of the models, characteristics of parameters contained therein, and given conditions. Therefore, one numerical technique is not sufficient to serve the general purpose, thereby leading many researchers to devise techniques each suitable for a particular set of problems and this search continues to this day.

Various scholars have either devised new numerical techniques to solve initial value problems or improved existing ones in many aspects such as convergence rate, order of accuracy, stability, efficiency, computational cost, number of slope evaluations per integration step, speed and implementation [11]. Standard numerical techniques to solve initial value problems in ordinary differential equations include linear explicit and implicit Runge-Kutta techniques, linear explicit and implicit Adams-Bashforth-Moulton schemes, exponential schemes, multiderivative schemes, backward differentiation formulae, and a few others [12]. Among the nonstandard; improved linear explicit Runge-Kutta schemes with reduced slope evaluations, accelerated Runge-Kutta schemes, singly-implicit Runge-Kutta schemes, A-stable Runge-Kutta collocation schemes, two-derivative Runge-Kutta schemes, semi-implicit hybrid schemes, explicit and implicit block schemes, and the list goes on as can be found in [13-17]. Apart from these, nonlinear/rational numerical techniques to solve mathematical models having characteristics of stiffness and singularity have been developed [18]. In addition to this, adaptive numerical techniques play 
their roles where the solution of the problem changes rapidly in some portions of the integration interval and slowly in others [19]. Apart from these, time fractional and partial differential equations are also of major importance as studied in [20-25].

\section{Derivation of the proposed scheme}

Consider a well-posed initial value problem of the form

$$
\begin{gathered}
\frac{d y(x)}{d x}=f(x, y(x)), y\left(x_{0}\right)=y_{0} \\
y, f(x, y(x)) \in \mathbb{R}, x \in[a, b] \subset \mathbb{R}
\end{gathered}
$$

This problem has been assumed to have a unique continuously differentiable solution $y(x)$ where $y\left(x_{n}\right) \approx y_{n}$ so that $y_{n}$ is considered to be approximation to the analytical (exact) solution $y(x)$ at $x=x_{n}$ along the integration interval $[a, b]$ with $h=\frac{|b-a|}{n}$ as a constant step-size used at per integration step.

Generally, a linear single-step explicit numerical algorithm to solve an initial value problem is given as:

$$
y_{n+1}=y_{n}+h \phi\left(x_{n}, y_{n} ; h\right)
$$

where $\phi_{f}\left(x_{n}, y_{n} ; h\right)$ can be expressed in terms of Taylor series expansion of an arbitrary function $f(x, y)$. Moreover, the Taylor series expansion of $y\left(x_{n}+h\right)$ is of the form:

$$
\begin{aligned}
& y\left(x_{n}+h\right)= \\
& y\left(x_{n}\right)+h f+\frac{1}{2 !} h^{2}\left(f_{x}+f f_{y}\right)+\frac{1}{3 !} h^{3}\left(f_{x x}+2 f f_{x y}+f^{2} f_{y y}+f f_{y}^{2}+f_{x} f_{y}\right) \\
& +\frac{1}{4 !} h^{4}\left(\begin{array}{l}
f_{x x x}+3 f f_{x x y}+3 f^{2} f_{x y y}+5 f f_{y} f_{x y}+3 f_{x} f_{x y}+f^{3} f_{y y y} \\
+4 f^{2} f_{y} f_{y y}+3 f f_{x} f_{y y}+f f_{y}^{3}+f_{x} f_{y}^{2}+f_{x x} f_{y}
\end{array}\right)+\mathscr{O}\left(h^{5}\right)
\end{aligned}
$$

The proposed scheme of the present study has been generally set up as

$$
y_{n+1}=y_{n}+h \varphi_{f}\left(x_{n}, y_{n} ; h\right)
$$

where

$$
\begin{gathered}
\varphi_{f}\left(x_{n}, y_{n} ; h\right)=b_{1} k_{1}+b_{2} k_{2}+b_{3} k_{3} \\
k_{1}=f\left(x_{n}, y_{n}\right), \quad k_{2}=f\left(x_{n}+a_{2} h, y_{n}+h k_{1}\left(b_{21}+h c_{21} f_{y}\right)\right) \\
k_{3}=f\left(x_{n}+a_{3} h, y_{n}+h\left(b_{31} k_{1}+b_{32} k_{2}\right)+h^{2} c_{31} k_{1} f_{y}\right)
\end{gathered}
$$


Expanding $k_{2}$ and $k_{3}$ in Taylor's series, we obtain

$$
\begin{gathered}
k_{2}= \\
f+\left(f f_{y} b_{21}+f_{x} a_{2}\right) h \\
+\left(\frac{1}{2} f_{y y} f^{2} b_{21}^{2}+f_{x y} f a_{2} b_{21}+f f_{y}^{2} c_{21}+\frac{1}{2} f_{x x} a_{2}^{2}\right) h^{2} \\
+\left(\frac{1}{6} f_{y y y} f^{3} b_{21}^{3}+\frac{1}{2} f_{x y y} f^{2} a_{2} b_{21}^{2}+f_{y y} f^{2} f_{y} b_{21} c_{21}\right. \\
\left.+\frac{1}{2} f_{x x y} f a_{2}^{2} b_{21}+f_{x y} f f_{y} a_{2} c_{21}+\frac{1}{6} f_{x x x} a_{2}^{3}\right) h^{3}+\mathscr{O}\left(h^{4}\right)
\end{gathered}
$$

$k_{3}=$

$$
\begin{aligned}
& f+\left(f_{y} f b_{32}+f_{y} f b_{31}+f_{x} a_{3}\right) h+\left(\begin{array}{l}
\frac{1}{2} f_{y y} f^{2} b_{32}^{2}+f_{y y} f^{2} b_{31} b_{32}+ \\
\frac{1}{2} f_{y y} f^{2} b_{31}^{2}+f_{x y} f a_{3} b_{32}+ \\
f_{x y} f a_{3} b_{31}+f_{y}^{2} f_{31}+\frac{1}{2} f_{x x} a_{3}^{2}+ \\
f_{y}^{2} f b_{21} b_{32}+f_{x} f_{y} a_{2} b_{32}
\end{array}\right) h^{2} \\
& +\left(\begin{array}{l}
\frac{1}{2} f_{y y y} f^{3} b_{31} b_{32}^{2}+\frac{1}{2} f_{y y y} f^{3} b_{31}^{2} b_{32}+\frac{1}{2} f_{x y y} f^{2} a_{3} b_{32}^{2}+\frac{1}{2} f_{x y y} f^{2} a_{3} b_{31}^{2}+ \\
\frac{1}{2} f_{x x y} f a_{3}^{2} b_{32}+\frac{1}{2} f_{x x y} f a_{3}^{2} b_{31}+\frac{1}{2} f_{y} f^{2} f_{y y} b_{21}^{2} b_{32}+f_{y}^{3} f b_{32} c_{21}+ \\
f_{y} f f_{x y} a_{2} b_{21} b_{32}+\frac{1}{2} f_{y} f_{x x} a_{2}^{2} b_{32}+\frac{1}{6} f_{y y y} f^{3} b_{32}^{3}+\frac{1}{6} f_{y y y} f^{3} b_{31}^{3}+ \\
f_{x y y} f^{2} a_{3} b_{31} b_{32}+f_{y y} f^{2} f_{y} b_{31} c_{31}+f_{x y} f f_{y} a_{3} c_{31}+f_{y} f^{2} f_{y y} b_{21} b_{31} b_{32}+ \\
f_{x} f f_{y y} a_{2} b_{31} b_{32}+\frac{1}{6} f_{x x x} a_{3}^{3}+f_{y} f^{2} f_{y y} b_{21} b_{32}^{2}+f_{x} f f_{y y} a_{2} b_{32}^{2}+ \\
f_{y} f f_{x y} a_{3} b_{21} b_{32}+f_{x} f_{x y} a_{2} a_{3} b_{32}+f_{y y} f^{2} f_{y} b_{32} c_{31}
\end{array}\right) h^{3}+\mathscr{O}\left(h^{4}\right)
\end{aligned}
$$

Substituting the result of $k_{1}, k_{2}$ and $k_{3}$ into (4) and then equating the coefficients of powers of $h$ up to $h^{3}$ with that of (3), one obtains the following conditions:

$$
\begin{array}{ccc}
b_{1}+b_{2}+b_{3}=1 & a_{2} b_{3} b_{32}=\frac{1}{6} & a_{2} b_{2}+a_{3} b_{3}=\frac{1}{2} \\
\frac{1}{2}\left(a_{2}^{2} b_{2}+a_{3}^{2} b_{3}\right)=\frac{1}{6} & b_{2} b_{21}+b_{3} b_{31}+b_{3} b_{32}=\frac{1}{2} & a_{2} b_{2} b_{21}+a_{3} b_{3} b_{31}+a_{3} b_{3} b_{32}=\frac{1}{3} \\
b_{2} c_{21}+b_{3} c_{31}+b_{3} b_{21} b_{32}=\frac{1}{6} & \frac{1}{2}\left(b_{2} b_{21}^{2}+b_{3} b_{31}^{2}+b_{3} b_{32}^{2}\right)+b_{3} b_{31} b_{32}=\frac{1}{6}
\end{array}
$$

This is a non-linear system of 8 equations and 10 unknowns which we have solved using MATLAB R2017a to obtain one of its optimal solutions which then formed the 
following third order numerical scheme:

$$
\begin{gathered}
k_{1}=f\left(x_{n}, y_{n}\right), \\
k_{2}=f\left(x_{n}+\frac{2}{3} h, y_{n}+\frac{2}{3} h k_{1}+\frac{1}{4} h^{2} k_{1} f_{y}\right), \\
k_{3}=f\left(x_{n}+\frac{2}{3} h, y_{n}-\left(\frac{1}{12} k_{1}-\frac{3}{4} k_{2}\right) h-\frac{5}{16} h^{2} k_{1} f_{y}\right), \\
y_{n+1}=y_{n}+\frac{1}{12} h\left(3 k_{1}+5 k_{2}+4 k_{3}\right)
\end{gathered}
$$

This is the proposed numerical scheme with third order accuracy.

\section{Local truncation error analysis}

In order to obtain the local truncation error of the proposed scheme, a usual functional associated to the scheme has been considered and is given below:

$$
\mathscr{L}(z(x), h)=z(x+h)-y_{n+1}
$$

where $z(x)$ is an arbitrary function defined along the integration interval $\left[x_{0}, x_{n}\right]$ and differentiable as many times as required. Having expanded it into the Taylor series about $x$ and collecting the terms in $h$, the local truncation error under local assumption of the following form has been obtained that ensures at least third order accuracy of the proposed scheme:

$T_{n}=\left(\begin{array}{c}-\frac{1}{48} f_{y}^{3} f-\frac{1}{72} f_{x x} f_{y}+\frac{1}{24} f_{x} f_{y}^{2}+\frac{1}{72} f_{x x y} f+\frac{1}{216} f_{y y y} f^{3} \\ +\frac{1}{72} f_{x y y} f^{2}+\frac{1}{72} f_{x y} f_{x}+\frac{1}{216} f_{x x x}-\frac{1}{72} f_{x y} f_{y} f+\frac{1}{72} f_{y y} f_{x} f\end{array}\right) h^{4}+\mathscr{O}\left(h^{5}\right)(6)$

\section{Consistency analysis}

Definition 4.1 Given an initial value problem $y^{\prime}(x)=f\left(x_{n}, y_{n}\right) ; y\left(x_{0}\right)=y_{0}$; an iterative scheme with an increment function $\Phi\left(x_{n}, y_{n} ; h\right)$ is said to be consistent if

$$
\lim _{h \rightarrow 0} \Phi\left(x_{n}, y_{n} ; h\right)=f\left(x_{n}, y_{n}\right)
$$

The increment function of the proposed scheme (7) is shown as:

$$
\begin{aligned}
\lim _{h \rightarrow 0} \Phi\left(x_{n}, y_{n} ; h\right) & =\frac{1}{12} \lim _{h \rightarrow 0}\left(3 k_{1}+5 k_{2}+4 k_{3}\right) \\
& =\frac{1}{12} \lim _{h \rightarrow 0}\left[\begin{array}{c}
3 f\left(x_{n}, y_{n}\right)+5 f\left(x_{n}+\frac{2}{3} h, y_{n}+\frac{2}{3} h k_{1}+\frac{1}{4} h^{2} k_{1} f_{y}\right)+ \\
4 f\left(x_{n}+\frac{2}{3} h, y_{n}-\left(\frac{1}{12} k_{1}-\frac{3}{4} k_{2}\right) h-\frac{5}{16} h^{2} k_{1} f_{y}\right)
\end{array}\right] \\
& =
\end{aligned}
$$


Thus, the proposed scheme is shown to be consistent with at least third order accuracy.

\section{Stability analysis}

For a numerical scheme to be reliable, it is important for the scheme to be stable. All this means is that the numerical error involved in the scheme should not grow without bound either as the solution of the initial value problem progresses or the step-size is decreased. In order to analyze the stability of the numerical scheme, one has numerous options with testing of the scheme on the differential equation having known exact well-defined solution being one of such options. Following the simple initial value problem is the standard numerical experiment for the stability analysis

$$
\frac{d y(x)}{d x}=\alpha y(x), y(0)=y_{0}, \alpha \in \mathbb{C}
$$

where the real part of $\alpha$ should lie on the left half of the complex plane $\mathbb{C}$.

Using slopes of the proposed scheme, one obtains

$$
\begin{gathered}
k_{1}=\alpha y_{n}, \\
k_{2}=\alpha y_{n}\left[1+\frac{2}{3} h \alpha+\frac{1}{4} h^{2} \alpha^{2}\right], \\
k_{3}=\alpha y_{n}\left[1+\frac{2}{3} h \alpha+\frac{3}{16} h^{2} \alpha^{2}+\frac{3}{16} h^{3} \alpha^{3}\right]
\end{gathered}
$$

Now upon using the proposed scheme on the test problem, the stability function $\Theta(z)$ has been found and the stability region (red) plotted in Figure 1.

$$
\Theta_{\text {Proposed }}(z)=1+z+\frac{1}{2} z^{2}+\frac{1}{6} z^{3}+\frac{1}{16} z^{4}, \quad \text { where } \quad z=h \alpha .
$$

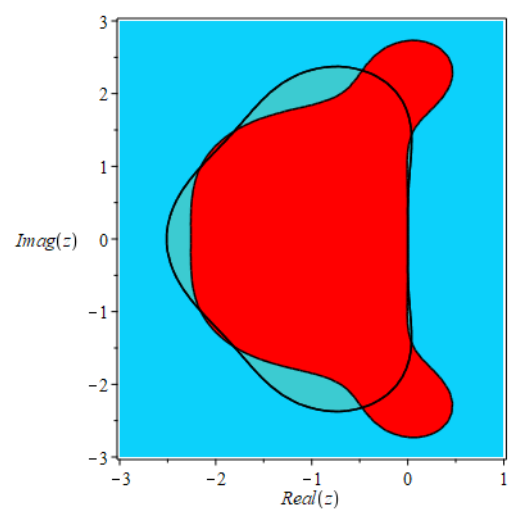

Fig. 1. Stability region of all three schemes with red for the proposed numerical scheme

In addition to this, the stability functions for third order Heun and Ralston schemes considered for comparative analysis are available from the relevant literature [1] and 
it can easily be observed that the stability function for the proposed numerical scheme goes to five-term approximation for the exponential function $\exp (z)$ with a minor difference in the coefficient of the fifth term whereas rest of the two schemes to four-term approximation as shown below:

$$
\Theta_{\text {Ralston, Heun }}(z)=1+z+\frac{1}{2} z^{2}+\frac{1}{6} z^{3}, \quad \text { where } \quad z=h \alpha .
$$

Moreover, it may also be noted that the proposed numerical scheme is convergent on the basis of being both stable and consistent as stated by the Dahlquist Equivalence Theorem in [1].

\section{Numerical experiments}

We have selected as many as four initial value problems of different natures (linear, nonlinear, autonomous and non-autonomous) in order to compare the efficiency of the proposed scheme against two well-known standard numerical schemes of third order accuracy, namely, Heun's and Ralston's schemes whose Butcher's tableau is given below. The performance of the new scheme has been checked with respect to the CPU time (seconds), maximum absolute relative error $E_{\max }=$ $=\max _{a \leq x \leq b}\left|\frac{y\left(x_{n}\right)-y_{n}}{y\left(x_{n}\right)}\right|$ and the absolute relative error found at the final grid point $E(x=b)=\left|\frac{y(b)-y_{x=b}}{y(b)}\right|$ of the integration interval used for the solution of initial value problems under consideration. Furthermore, in order to test the third accuracy of the proposed scheme for the selected initial value problems; these problems have been numerically solved by the proposed scheme by decreasing one order of magnitude in the step-size and it is observed in the Table 5 that by doing so there is a drop of three orders of magnitude in the maximum absolute relative error computed over the corresponding integration interval thereby supporting the claim of third order accuracy of the proposed scheme.

\begin{tabular}{|c|c|c|c|c|c|c|c|}
\hline \multicolumn{4}{|c|}{ Heun } & \multicolumn{4}{|c|}{ Ralston } \\
\hline 0 & 0 & 0 & 0 & 0 & 0 & 0 & 0 \\
\hline $1 / 3$ & $1 / 3$ & 0 & 0 & $1 / 2$ & $1 / 2$ & 0 & 0 \\
\hline $2 / 3$ & 0 & $2 / 3$ & 0 & 1 & -1 & 2 & 0 \\
\hline & $1 / 4$ & 0 & $3 / 4$ & & $1 / 6$ & $2 / 3$ & $1 / 6$ \\
\hline
\end{tabular}

Problem 1. $\frac{d y}{d x}=10 x y(x) ; y(0)=1,0 \leq x \leq 1$.

Analytical Solution: $y(x)=\exp \left(5 x^{2}\right)$.

Problem 2. $\frac{d y}{d x}=x y(x)^{2}-y(x) ; y(0)=1,0 \leq x \leq 1$.

Analytical Solution: $y(x)=\frac{1}{x+1}$.

Problem 3. $\frac{d y}{d x}=1+\frac{5 y(x)}{x+1} ; \quad y(0)=1,0 \leq x \leq 2$. 
Analytical Solution: $y(x)=-\frac{1}{4}-\frac{1}{4} x+\frac{5}{4}(1+x)^{5}$.

Problem 4. $\frac{d y}{d x}=y(x)^{2} \sinh (x) ; y(0)=1,0 \leq x \leq 1$.

Analytical Solution: $y(x)=-\frac{1}{(\cosh (x)-2)}$.

Table 1. CPU Times, Max. Absolute Relative Errors over [0,1] and Absolute Relative Errors at $x=1$ for Problem 1 with $h=0.01$

\begin{tabular}{|l|l|l|l|}
\hline \multicolumn{1}{|c|}{ Scheme } & CPU Time & Max. Error & Last Error \\
\hline New & $8.9530 \mathrm{e}-03$ & $2.6059 \mathrm{e}-05$ & $2.6059 \mathrm{e}-05$ \\
\hline Heun & $4.0807 \mathrm{e}-03$ & $1.0508 \mathrm{e}-04$ & $1.0508 \mathrm{e}-04$ \\
\hline Ralston & $4.2522 \mathrm{e}-03$ & $7.4192 \mathrm{e}-05$ & $7.4192 \mathrm{e}-05$ \\
\hline
\end{tabular}

Table 2. CPU Times, Max. Absolute Relative Errors over $[0,1]$ and Absolute Relative Errors at $x=1$ for Problem 2 with $h=0.01$

\begin{tabular}{|l|l|l|l|}
\hline \multicolumn{1}{|c|}{ Scheme } & CPU Time & Max. Error & Last Error \\
\hline New & $6.9974 \mathrm{e}-04$ & $2.4143 \mathrm{e}-08$ & $2.4143 \mathrm{e}-08$ \\
\hline Heun & $5.0111 \mathrm{e}-04$ & $7.0045 \mathrm{e}-08$ & $7.0045 \mathrm{e}-08$ \\
\hline Ralston & $4.9688 \mathrm{e}-04$ & $4.4909 \mathrm{e}-08$ & $4.4909 \mathrm{e}-08$ \\
\hline
\end{tabular}

Table 3. CPU Times, Max. Absolute Relative Errors over [0,2] and Absolute Relative Errors at $x=2$ for Problem 3 with $h=0.01$

\begin{tabular}{|l|l|l|l|}
\hline \multicolumn{1}{|c|}{ Scheme } & CPU Time & Max. Error & Last Error \\
\hline New & $5.9711 \mathrm{e}-04$ & $4.8201 \mathrm{e}-06$ & $4.8201 \mathrm{e}-06$ \\
\hline Heun & $2.5237 \mathrm{e}-04$ & $5.3936 \mathrm{e}-06$ & $5.3936 \mathrm{e}-06$ \\
\hline Ralston & $3.2723 \mathrm{e}-04$ & $7.8033 \mathrm{e}-06$ & $7.8033 \mathrm{e}-06$ \\
\hline
\end{tabular}

Table 4. CPU Times, Max. Absolute Relative Errors over $[0,1]$ and Absolute Relative Errors at $x=1$ for Problem 4 with $h=0.01$

\begin{tabular}{|l|l|l|l|}
\hline Scheme & CPU Time & Max. Error & Last Error \\
\hline New & $2.8980 \mathrm{e}-04$ & $1.3278 \mathrm{e}-07$ & $6.4499 \mathrm{e}-08$ \\
\hline Heun & $1.1049 \mathrm{e}-04$ & $3.0137 \mathrm{e}-06$ & $3.0137 \mathrm{e}-06$ \\
\hline Ralston & $1.0203 \mathrm{e}-04$ & $1.5386 \mathrm{e}-07$ & $7.0364 \mathrm{e}-09$ \\
\hline
\end{tabular}

Table 5. A drop of three orders of magnitude in the maximum absolute relative error for every one drop of magnitude in the step-size $h$ over the corresponding integration interval for Problems 1-4 using the proposed scheme

\begin{tabular}{|l|l|l|l|}
\hline Problem $\backslash h$ & 0.01 & 0.001 & 0.0001 \\
\hline 1 & $1.3278 \mathrm{e}-07$ & $1.2194 \mathrm{e}-10$ & $1.8328 \mathrm{e}-13$ \\
\hline 2 & $2.4143 \mathrm{e}-08$ & $2.4266 \mathrm{e}-11$ & $6.4834 \mathrm{e}-14$ \\
\hline 3 & $4.8201 \mathrm{e}-06$ & $4.9965 \mathrm{e}-09$ & $5.1997 \mathrm{e}-12$ \\
\hline 4 & $2.6059 \mathrm{e}-05$ & $3.0496 \mathrm{e}-08$ & $3.0570 \mathrm{e}-11$ \\
\hline
\end{tabular}




\section{Results and discussions}

The initial value problems originating from applied and computational mathematics can be dealt with using the new third order proposed scheme described in this paper. Four problems of a different nature have been numerically solved by the scheme and compared with two standard numerical schemes (Heun and Ralston) taken from the relevant literature. It may be observed from the Tables 1-4 that the maximum absolute relative error produced by the proposed scheme is about four-fold and thrice smaller than Heun's and Ralston's schemes respectively in test problem 1 and for the rest of the problems, the proposed scheme follows nearly the same trend while consuming almost a little large amount of time on average. In addition, the new scheme has the behavior of decreasing errors with an increase in the number of integration steps, and a similar type of behavior can be depicted by the remaining four test problems. Hence, it would be safe to claim that the new proposed scheme is superior to Heun's and Ralston's third order schemes in terms of convergence. Not only this, the new scheme may well be compared with any existing numerical scheme having the same order of local accuracy as that of the proposed scheme itself.

\section{Conclusions}

In this paper, a new numerical scheme has been developed that uses three slopes per integration step and maintains the third order accuracy as shown above using the idea of a local truncation error in terms of Taylor series expansion. The new scheme is capable of solving initial value problems arising in various fields of science and engineering. Derivation of the scheme is followed by its error analysis, consistency and stability wherein it shows overall satisfactory performance. The stability region shown by Figure 1 reveals that the new scheme is conditionally stable like every other existing linear explicit numerical scheme in the relevant literature. The test problems under consideration have well been solved by the new scheme and by other two schemes having same order of accuracy where it is easy to observe from above tables that the new scheme produces smaller errors whether it be a maximum absolute relative error or the absolute relative error computed at the last grid point of the associated interval of integration. Thus, the new proposed scheme can reliably be employed to solve initial value problems in ordinary differential equations in replacement of any other existing third order linear explicit numerical scheme.

\section{Acknowledgment}

The authors are highly thankful to anonymous reviewer(s) for the constructive criticism and suggestions to improve the first version of this research paper. Furthermore, the first and third authors are grateful to the Mehran University of Engineering and Technology, Jamshoro for providing the facilities. 


\section{References}

[1] Butcher, J.C. (2016). Numerical Methods for Ordinary Differential Equations. John Wiley \& Sons.

[2] Strogatz, S.H. (2018). Nonlinear Dynamics and Chaos: With Applications to Physics, Biology, Chemistry, and Engineering. CRC Press.

[3] Sundnes, J., Lines, G.T., \& Tveito, A. (2001). Efficient solution of ordinary differential equations modeling electrical activity in cardiac cells. Mathematical Biosciences, 172(2), 55-72.

[4] Jordan, D.W., \& Smith, P. (1999). Nonlinear ordinary differential equations: an introduction to dynamical systems (Vol. 2). Oxford University Press, USA.

[5] Tuck, E.O., \& Schwartz, L.W. (1990). A numerical and asymptotic study of some third-order ordinary differential equations relevant to draining and coating flows. SIAM Review, 32(3), 453-469.

[6] Qu, Z., \& Garfinkel, A. (1999). An advanced algorithm for solving partial differential equation in cardiac conduction. IEEE Transactions on Biomedical Engineering, 46(9), 1166-1168.

[7] Zill, D.G. (2012). A First Course in Differential Equations with Modeling Applications. Cengage Learning.

[8] Hertz, J.A. (2018). Introduction to the Theory of Neural Computation. CRC Press.

[9] Lobo, J.Z., \& Valaulikar, Y.S. (2019). Lie symmetries of first order neutral differential equations. Journal of Applied Mathematics and Computational Mechanics, 18(1), 29-40.

[10] Fedorenko, R.P. (1994). Stiff systems of ordinary differential equations. Numerical Methods and Applications, 117-154.

[11] Zill, D., Wright, W.S., \& Cullen, M.R. (2011). Advanced Engineering Mathematics. Jones \& Bartlett Learning.

[12] Chapra, S.C. (2012). Applied Numerical Methods. Columbus: McGraw-Hill.

[13] Yahya, N.A. (2017). Semi-implicit two-step hybrid method with FSAL property for solving second-order ordinary differential equations. International Journal of Advanced and Applied Sciences, 4(6), 169-174.

[14] Udwadia, F.E., \& Farahani, A. (2008). Accelerated Runge-Kutta methods. Discrete Dynamics in Nature and Society, 2008.

[15] Aliya, T., Shaikh, A.A., \& Qureshi, S. (2018). Development of a nonlinear hybrid numerical method. Advances in Differential Equations and Control Processes, 19(3), 275-285.

[16] Qureshi, S., Memon, Z., \& Shaikh, A.A. (2018). Local accuracy and error bounds of the improved Runge-Kutta numerical methods. Journal of Applied Mathematics and Computational Mechanics, 17(4).

[17] Qureshi, S., \& Emmanuel, F.S. (2018). Convergence of a numerical technique via interpolating function to approximate physical dynamical systems. Journal of Advanced Physics, 7(3), 446-450.

[18] Ramos, H., Kalogiratou, Z., Monovasilis, T., \& Simos, T.E. (2016). An optimized two-step hybrid block method for solving general second order initial-value problems. Numerical Algorithms, 72(4), 1089-1102.

[19] Qureshi, S., \& Ramos, H. (2018). L-stable explicit nonlinear method with constant and variable step-size formulation for solving initial value problems. International Journal of Nonlinear Sciences and Numerical Simulation, 19(7-8), 741-751.

[20] Arqub, O.A., \& Al-Smadi, M. (2018). Atangana-Baleanu fractional approach to the solutions of Bagley-Torvik and Painlevé equations in Hilbert space. Chaos, Solitons \& Fractals, 117, 161-167.

[21] Osman, M.S. (2019). One-soliton shaping and inelastic collision between double solitons in the fifth-order variable-coefficient Sawada-Kotera equation. Nonlinear Dynamics, 96, 1491-1496. 
[22] Abu Arqub, O. (2019). Application of residual power series method for the solution of timefractional Schrödinger equations in one-dimensional space. Fundamenta Informaticae, 166(2), $87-110$.

[23] Ali, M.N., Osman, M.S., \& Husnine, S.M. (2019). On the analytical solutions of conformable time-fractional extended Zakharov-Kuznetsov equation through $\left(G^{\prime} / G^{2}\right)$-expansion method and the modified Kudryashov method. SeMA Journal, 76(1), 15-25.

[24] Abu Arqub, O. (2019). Numerical algorithm for the solutions of fractional order systems of Dirichlet function types with comparative analysis. Fundamenta Informaticae, 166(2), 111-137.

[25] Lu, D., Osman, M.S., Khater, M.M.A., Attia, R.A.M., \& Baleanu, D. (2020). Analytical and numerical simulations for the kinetics of phase separation in iron $(\mathrm{Fe}-\mathrm{Cr}-\mathrm{X}(\mathrm{X}=\mathrm{Mo}, \mathrm{Cu}))$ based on ternary alloys. Physica A: Statistical Mechanics and its Applications, 537, 122634. 